\title{
One Step Synthesis, Characterization of Modified Montmorillonite with Hydrothermal-Assist for Triazolone Sustained Release System with pH Sensitivity
}

\author{
Hua XU, Hongkai DENG, Huayao CHEN, Hongjun ZHOU *, Xinhua ZHOU, Xia PU
}

Key Laboratory of Agricultural Green Fine Chemicals of Guangdong Higher Education Institution, School of Chemistry and Chemical Engineering, Zhongkai University of Agriculture and Engineering, No.24, Dongsha Street, Haizhu District, GuangzhouCity, Guangdong, P.R.China

crossref http://dx.doi.org/10.5755/j01.ms.26.4.22019

\section{Received 02 January 2019; accepted 08 January 2019}

\begin{abstract}
Montmorillonite K-10 clay (MMT) was surface-modified by sodium dodecyl sulfate (SDS) and grafted with KH-550 coupling agent to construct "Hy-MMT" organic modified structure in one step with hydrothermal-assist. Different sustained release systems were prepared through changing the temperature during the hydrothermal process. The characterization results illustrated the mechanism of the hydrothermal assist in intercalation during the organic modification. It was also proved that the triazolone loaded in the sustained release system existed as homogeneous in the pores as amorphous state by DSC analysis. According to SEM and TEM observation, the surface of original MMT or modified MMT without hydrothermal assist presented rough particles, which belonged to the SDS and KH-550 without intercalation into the layer structure which is in accordance with the contact angles of the samples. The experimental results indicated that the adsorption capacity (AC) of Hy-60-MMT showed the best due to its mild react condition the successfully intercalated by SDS and KH-550 by the assist of the hydrothermal treatment. Its AC for triazolone was significantly increased to $29.8 \mathrm{mg} / \mathrm{g}$ comparing to MMT without modification which showed no capacity for triazolone adsorption. The as-synthesized system also showed significant $\mathrm{pH}$ sensitivity with a sequence of $\mathrm{pH}=7>\mathrm{pH}=3>\mathrm{pH}=5>\mathrm{pH}=9$ in release speed indicating that the sustained release system can be well stored in mild acid or basic environment and activated while sprayed into earth or plants under neutral condition.

Keywords: montmorillonite, hydrothermal-assist, $\mathrm{KH}-550$, triazolone, sustained release.
\end{abstract}

\section{INTRODUCTION}

Nowadays, the materials with ordered pore structure have drawn significant interest [1,2]. Among them, montmorillonite (MMT) with layered pores has high capacity, high surface area, high porosity, low cost and better surface activity [3], due to which it is a widely used adsorbent for treatment of toxic organic pollutants in water purification and remediation of industrial wastes $[4,5]$. In order to better utilization of MMT which must be organic treatment because montmorillonite has a hydrophilic oleophobic properties and compatibility with the organic material is poor [6]. And the nanocomposites of modified clays are known to exhibit remarkable improvement in strength/heat resistance, decrease in gas permeability/flammability, and increase in biodegradability [3] compared to their untreated counterparts.

Hydrothermal synthesis allows excellent control over particle size, shape, distribution and crystallinity of material [7]. The synthesis is conducted in a stainless autoclave using water as a solvent and nanoplates formation process occurs under high autogenous pressure at a certain synthesis temperature. Therefore, Hydrothermal has many advantages like low reaction temperature and easy variation of synthesis conditions including temperature, pressure, $\mathrm{pH}$, atmosphere etc. [8]. $\mathrm{Ai}$ and $\mathrm{Li}$ [9] produced MMT-hydrochar nanocomposites by hydrothermal process and evaluated their performances

\footnotetext{
* Corresponding author. Tel.: +86-020-89003114; fax: +86-02089003114. E-mail address: hongjunzhou@163.com (H. Zhou)
}

in the adsorptive removal of organic dyes (methylene blue) in aqueous solution. Yang et al. [10] discussed the interaction of cellulose and montmorillonite in a hydrothermal process. In these studies, Organic matters can not only be adsorbed by MMt, and be immobilized at the surface, but also can enter the interlayer spaces of MMT. However, the study in this sustained released system based on modified MMT for the pesticide delivery was rarely reported.

In our previous researches, MCM-41 was prepared through sol-gel method and encapsulated by polydopamine (PDA) before coordinated with metal ion to form a highly efficient sustained release system (M-PDA-MCM-41) for triazolone delivery [11]. In controlled release method, not only the utilization of agricultural pesticides is maintained efficient for longer time but also it reduces the pollution of the environment [12]. But the expensive price of PDA and MCM-41 prevents its further application in the practical use. On the other side, MMT with low-cost and similar components of soil may be more attractive.

In this work, montmorillonite K-10 clay (MMT) was surface-modified by sodium dodecyl sulfate (SDS), which is selected as the co-intercalation agent with coupling agent $\mathrm{KH}-550$ explained in terms of the closeness of the SDS length $(18.0 \AA)$ in the long-axis direction to the MMT interlayer $(14.3 \AA)$, thus promoting the nanoenvironment and compositions of the tuneable interlayer [13] to construct "Hy-MMT" organic modified structure in one step [14]. Triazolone which are widely considered to be antibacterial, anti-inflammatory, antiviral, antitumour and antiasthmatic agents [15] was used as a model drug and 
loaded via impregnation construct Tri/Hy-MMT sustained release system. A silane coupling agent (KH-550), which could react with both the hydroxyl groups of MMT and coordinated with triazolone with its amino group, was recommended to connect them $[16,17]$. The relationship between hydrothermal preparation and sustained release performance was investigated. Finally, the highly efficient sustained release system with $\mathrm{pH}$-sensitivity for pesticide delivery was developed, which not only maintain the utilization of agricultural pesticides efficient for longer time but also reduces the pollution of the environment.

\section{MATERIALS AND METHODS}

\subsection{Materials}

Montmorillonite $\mathrm{K}-10$ clay (MMT) and $\mathrm{KH}-550$ (molecular formula $\left.\mathrm{NH}_{2}\left(\mathrm{CH}_{2}\right)_{3} \mathrm{Si}\left(\mathrm{OC}_{2} \mathrm{H}_{5}\right)_{3}\right)$ were obtained from Macklin. Ethanol, sodium dodecyl sulfate (SDS), sodium hydroxide and hydrochloride were obtained from Tianjin Damao Chemical Reagents. And triazolone (> $99 \%$, Jiangsu Jinghong Chemical Engineering Co., Ltd.) was also used in this work. All chemicals were analytical grade and used as received without any further purification. All solutions were prepared with distilled water.

\subsection{Sample preparation and characterization}

The One step preparation method was modified from the previous synthesis protocol $[16,18]$ described as follow, MMT (1 g) and KH-550 (0.7 g) was dispersed in a $30 \mathrm{~mL}$ SDS solution $(15 \mathrm{mg} / \mathrm{mL})$ of ethanol and water $(75 / 25)$ and then stirred vigorously for $2 \mathrm{~h}$ to make MMT disperse completely in the solvent at $40{ }^{\circ} \mathrm{C}$. Then the mixture solution was transferred into a $50 \mathrm{~mL}$ Teflon-lined stainless steel autoclave, and was hydrothermally treated under autogenic pressure at $60{ }^{\circ} \mathrm{C}, 80{ }^{\circ} \mathrm{C}$ and $100{ }^{\circ} \mathrm{C}$ respectively before being filtered, washed and dried in oven at $60^{\circ} \mathrm{C}$ for $24 \mathrm{~h}$ to obtain the products denoted as Hy-60-MMT, Hy-80-MMT and Hy-100-MMT. For comparison, 80-MMT was prepared as illustrated above but treated under atmospheric pressure at $80^{\circ} \mathrm{C}$ instead of hydrothermal process. Hy-s-80-MMT was prepared from mixing and the MMT after hydrothermal treatment at $80{ }^{\circ} \mathrm{C}$ and $\mathrm{KH}-550$ in SDS solution under atmospheric pressure at $40{ }^{\circ} \mathrm{C}$ then stirred vigorously for $2 \mathrm{~h}$ before filtered, washed and dried in oven at $60{ }^{\circ} \mathrm{C}$ for $24 \mathrm{~h}$.

The supported triazolone was prepared via impregnation, which has been illustrated in our previous research [11]. The samples were activated under vacuum at $80{ }^{\circ} \mathrm{C}$ for $6 \mathrm{~h}$. And $0.3 \mathrm{~g}$ of samples was immersed in $30 \mathrm{~mL}$ of triazolone ethanol solution $(10 \mathrm{mg} / \mathrm{mL})$ at $35^{\circ} \mathrm{C}$ under stirring for $24 \mathrm{~h}$, then filtered, washed, and dried. The samples obtained were denoted as Tri/Hy-s-80-MMT, Tri/80-MMT, Tri/Hy-60-MMT, Tri/Hy-80-MMT and Tri/Hy-100-MMT respectively according to the different carrier. And the filter liquor was characterized by UV-vis to calculate the concentration after adsorption.

The samples were analyzed using a Bruker AXS D8 $\mathrm{X}$-ray diffractometer (Bruker AXS GmbH, Karlsruhe, Germany) with $\mathrm{Cu}$ radiation $(\lambda=1.5418 \AA)$ and a graphite monochromator at $25^{\circ} \mathrm{C}, 40 \mathrm{kV}$, and $30 \mathrm{~mA}$. The measurements were scanned at $2 \%$ min (angular range $2^{\theta}=0.5 \sim 10^{\circ}$ ) in $0.02^{\circ}$ step size. The structure of the samples was analyzed by a Spectrum 100 Fourier infrared spectrometer (PerkinElmer Inc., USA) by using the $\mathrm{KBr}$ squash technique. A Q200 differential scanning calorimeter (TA Co., USA) was used to conduct differential scanning calorimetry and detect the crystalline degree of the triazolone in the particles over a heating range of $20 \sim 160{ }^{\circ} \mathrm{C}$ and a heating rate of $20^{\circ} \mathrm{C} / \mathrm{min}$. The gold particles were sprayed on the surface of calcined samples under protection of $\mathrm{N}_{2}$ and the calcined samples were characterized by an $\mathrm{S} 4800$ scanning electron microscope (Hitachi, Japan) to observe the surface topography. TEM observation was conducted on a FEI Tecnai G2 F20 transmission election microscope (FEI, USA). The contact angle was analysed by Theta contact angle analyser. BET surface area was determined by $\mathrm{N}_{2}$ adsorption isotherms at $-196{ }^{\circ} \mathrm{C}$, operated on Quadrasorb SI adsorption equipment. The samples were degassed at $200{ }^{\circ} \mathrm{C}$ for $12 \mathrm{~h}$ in vacuum before $\mathrm{N}_{2}$ adsorption experiment.

\subsection{Adsorption properties test}

A UV-2550 UV-Vis spectrophotometer from Shimadzu Co., Japan, was applied to measure the amount of triazolone adsorbed by MMT. Linear regression of the solution concentration $\left(\mathrm{C} /\left(\mathrm{mg} \times \mathrm{L}^{-1}\right)\right)$ and absorbance $(A)$ of triazolone standard ethanol solutions of different concentrations at $\lambda=223 \mathrm{~nm}$ was performed to obtain a standard curvilinear equation: $C=40.00 A+9.220$, $\mathrm{R}^{2}=0.9993$. UV spectroscopy was performed to measure the absorbance of this ethanol solution before and after the adsorption in triazonlone ethanol solution. Adsorption capacity $(A C)$ and loading content $(L C)$ may be calculated by the following equation:

$$
\begin{aligned}
& A C=\frac{\left(C_{0}-C_{1}\right) \times V}{m} ; \\
& L C=\frac{\left(C_{0}-C_{1}\right) \times V}{m \times 1000+\left(C_{0}-C_{1}\right) \times V},
\end{aligned}
$$

where $C_{0}$ is the origin mass concentration $(\mathrm{mg} / \mathrm{L})$ of the triazolone in ethanol solution; $C_{l}$ is the mass concentration $(\mathrm{mg} / \mathrm{L})$ of the triazolone in ethanol solution after adsorption, and $m$ is the mass ( $\mathrm{g}$ ) of samples.

\subsection{Sustained released performance test}

The performance of sustained-release triazolone particles was tested according to the reference [19]. The $\left(\mathrm{M}_{1}, \mathrm{mg}\right)$ drug-loaded particles were weighed and placed in a conical flask filled with $50 \mathrm{~mL}$ of $50 \%$ ethanol. Linear regression of the solution concentration $\left(\mathrm{C} /\left(\mathrm{mg} \times \mathrm{L}^{-1}\right)\right)$ and absorbance $(A)$ of triazolone standard solutions of different concentrations at $\lambda=223 \mathrm{~nm}$ was performed to obtain a standard curvilinear equation: $C=28.82 A+0.02248$, $\mathrm{R}^{2}=0.9993$. At intervals of $(\mathrm{t}), 1 \mathrm{~mL}$ of the sample solution was transferred and diluted to $25 \mathrm{~mL}$. An equal volume of the original sustained-release solution was then added to the conical flask to replace the withdrawn sample. The absorbance of the $25 \mathrm{~mL}$ solution was obtained, and the cumulative release amount of triazolone was calculated as 
$\mathrm{R}_{\mathrm{i}}$. A $\mathrm{R}_{\mathrm{i}}-\mathrm{t}$ curve was drawn to study the release kinetics of triazolone. $\mathrm{R}_{\mathrm{i}}$ may be calculated by the following equation:

$\mathrm{R}_{\mathrm{i}}=\left\{\begin{array}{l}\frac{\rho_{\mathrm{i}} \times 0.1}{\mathrm{M}_{1} \times L C}(i=1) \\ \frac{\rho_{\mathrm{i}} \times 0.1}{\mathrm{M}_{1} \times L C}+\frac{\sum_{i=1}^{i-1} \rho_{\mathrm{i}} \times 0.002}{\mathrm{M}_{1} \times L C}(i=2,3,4 \ldots)\end{array}\right.$,

where $\rho_{\mathrm{i}}$ is the mass concentration $(\mathrm{mg} / \mathrm{L})$ of triazolone for each sampling. Each datum was measured in triplicates, and the standard error was evaluated and presented with error bar in the figures.

\section{RESULTS AND DISCUSSION}

\subsection{Characterization of MMT}

FT-IR analysis was applied to examine the possible interaction components of the prepared samples as shown in Fig. 1. The absorption bands at 1631, 780 and $520 \mathrm{~cm}^{-1}$ were associated with the $-\mathrm{OH}$ stretching vibration, the $\mathrm{Al}$ $\mathrm{O}$ stretching vibration and the stretching mode of sixcoordinated Al originated from MMT, respectively $[20,21]$. The strong adsorption peak located at $1030 \mathrm{~cm}^{-1}$ corresponded to the stretching vibration of $\mathrm{Si}-\mathrm{O}$ in MMT [22]. After modification, two peaks observed at about $2863 \mathrm{~cm}^{-1}$ and $2930 \mathrm{~cm}^{-1}$ correspond to the methylene stretching vibration peak and methylene anti-symmetric stretching vibration peak from KH-550 [16]. The band at $627 \mathrm{~cm}^{-1}$ was associated with the in-plane bending vibration of $\mathrm{H}-\mathrm{N}-\mathrm{H}$ bond from $\mathrm{KH}-550$ [23]. And the characteristic bands at $1014 \mathrm{~cm}^{-1}$ assigned to the symmetric -SO stretching originated from sulfate ions of SDS can be clearly observed for SDS [7].

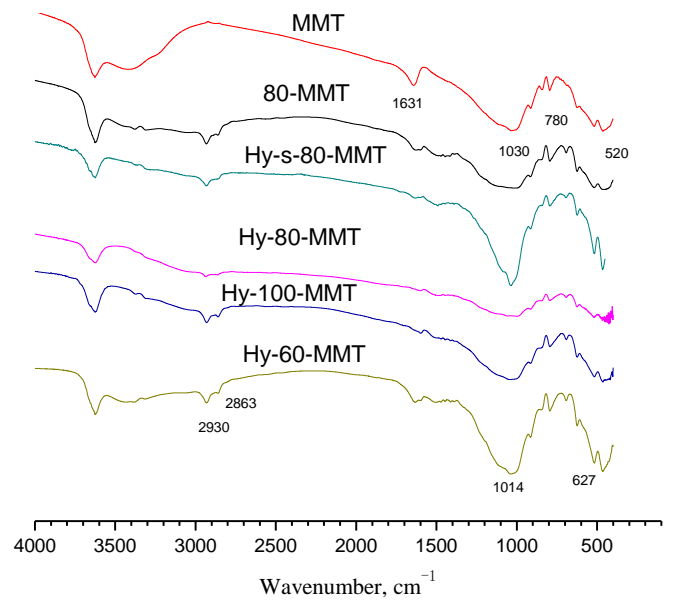

Fig. 1. FTIR spectra of MMT, 80-MMT, Hy-s-80-MMT, Hy-80MMT, Hy-100-MMT and Hy-60-MMT

Fig. 2 shows the XRD patterns of MMT before and after modification. As presented, the characteristic peaks of MMT are presented in all the samples, in which the main mineralogical composition $\mathrm{SiO}_{2}$ and $\mathrm{Al}_{2} \mathrm{O}_{3}$ of the samples were marked in the Fig. 2. There was a sharp characteristic peak in all samples ascribed to (001) crystal face, which characterized a layered structure and corresponded to the basal spacing with the higher reflection order. The d-spacing of the MMT, 80-MMT and
Hy-80-MMT were $1.43 \mathrm{~nm}, 1.06 \mathrm{~nm}$ and $1.06 \mathrm{~nm}$ respectively. The d-spacing of 80-MMT and Hy-80-MMT were decreased, indicating that the interlayer space was occupied by KH-550 and SDS.

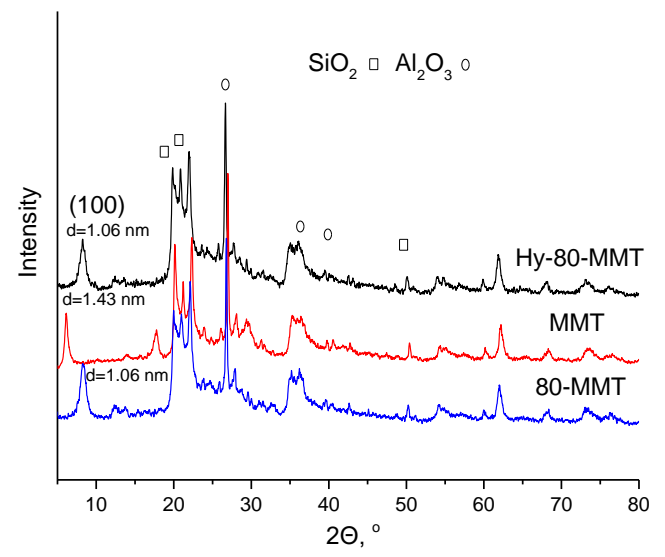

Fig. 2. XRD patterns of MMT, 80-MMT and Hy-80-MMT

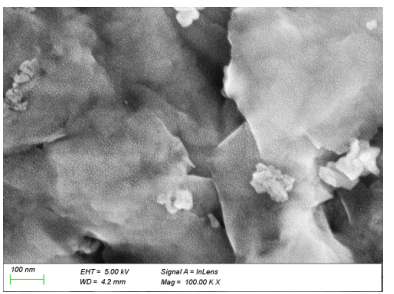

a

c

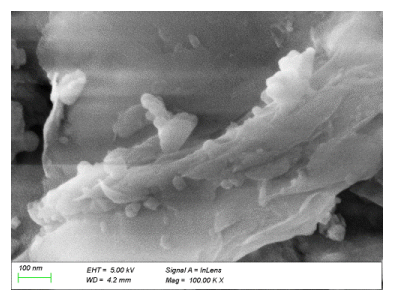

e

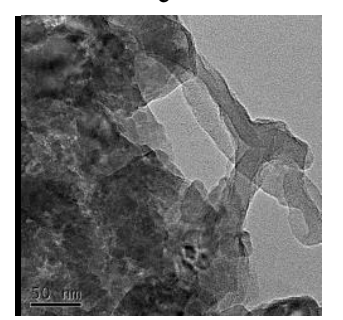

g

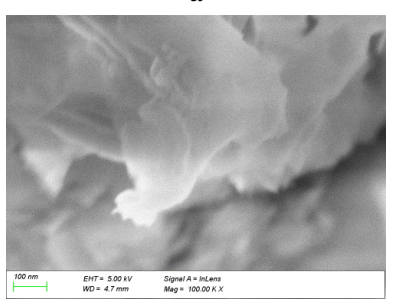

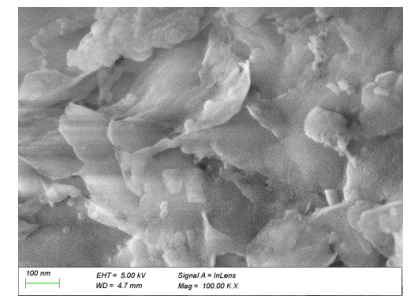

b

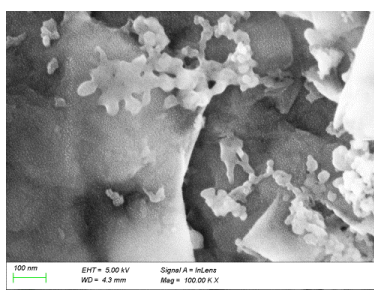

d

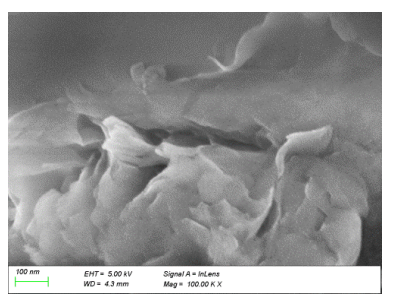

f

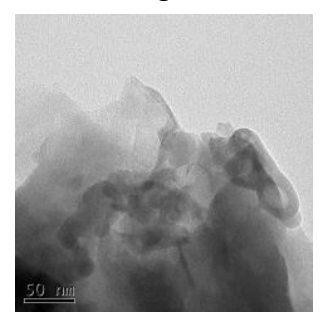

$\mathrm{h}$
Fig. 3. SEM image of: a-MMT; b-80-MMT; c-Hy-80-MMT; d-Hy-100-MMT; e-Hy-60-MMT; f-Tri/Hy-80-MMT; g-TEM image of 80-MMT; $\mathrm{h}-\mathrm{Hy}-60-\mathrm{MMT}$

As shown, the surface of 80-MMT (Fig. 3 b) and Hy100-MMT (Fig. $3 \mathrm{~d}$ ) particles presented rough particles which belonged to the SDS and KH-550 without intercalation into the layer structure. On the contrary, the 
surface of Hy-80-MMT (Fig. 3 c), Hy-60-MMT (Fig. 3 e) and Tri/Hy-80-MMT presented smooth layers even after loading triazolone. And comparing the TEM image of 80MMT with Hy-60-MMT shown in Fig. $3 \mathrm{~g}$ and $\mathrm{h}$, the rough particles in the surface of the samples disappeared after hydrothermal treatment in according with SEM imges which indicated the SDS and KH-550 were successfully intercalated into the layer structure and organic modified structure was created. What's more, the contact angle of MMT, 80-MMT, Hy-60-MMT. Hy-80-MMT and Hy-100MMT were 39.0, 36.6, 45.0, 44.6 and 35.2 respectively as shown in Fig. 4 indicating the SDS and KH-550 intercalation increased the hydrophobicity of the samples.

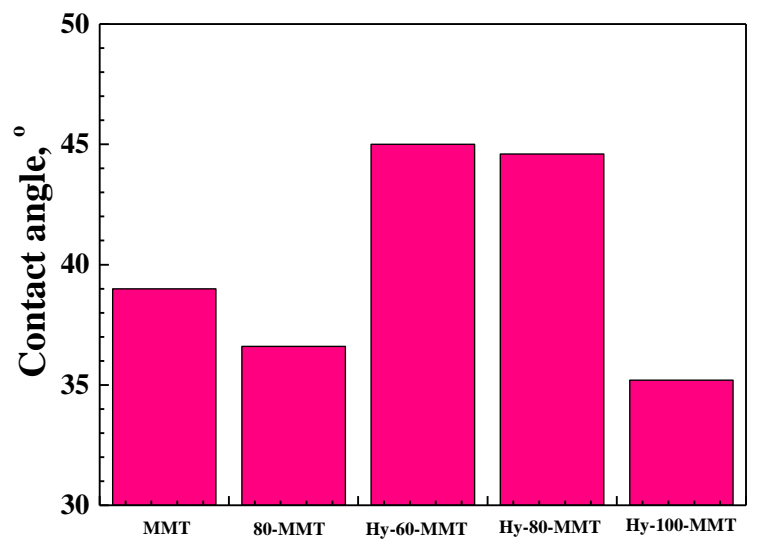

Fig. 4. The contact angle of MMT, 80-MMT, Hy-60-MMT. Hy80-MMT, and Hy-100-MMT

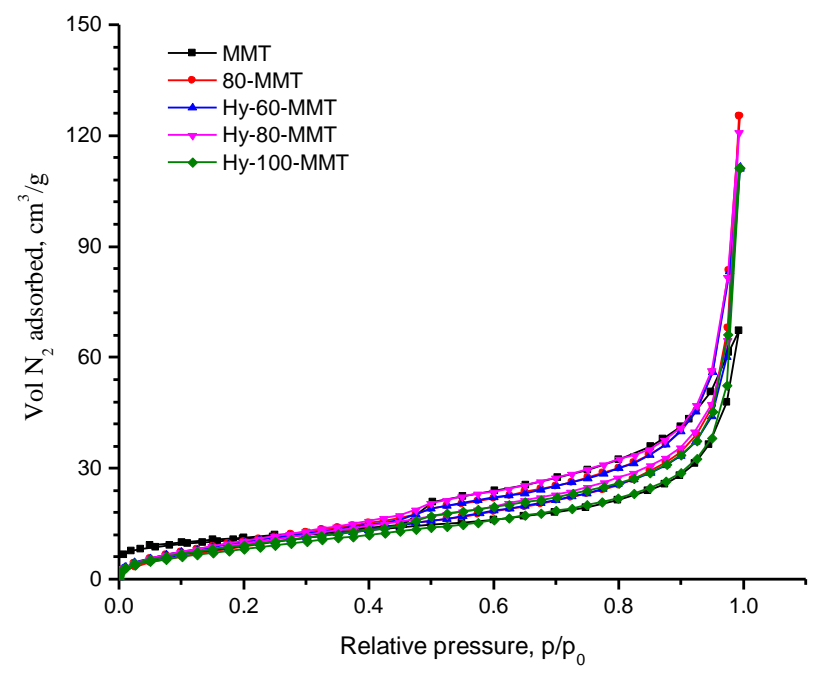

Fig. 5. $\mathrm{N}_{2}$ adsorption/desorption isotherms of MMT, 80-MMT, Hy-60-MMT. Hy-80-MMT, and Hy-100-MMT

As shown in Fig. 5, the $\mathrm{N}_{2}$ adsorption/desorption isotherms of MMT, 80-MMT, Hy-60-MMT. Hy-80-MMT, and Hy-100-MMT belong to type- II with $\mathrm{H} 3$ hysteresis loop. And the BET surface and pore data were calculated by Multi-Point BET and BJH desorption method respectively. And the hysteresis loop still remained after intercalation for Hy-60-MMT, Hy-80-MMT and Hy-100MMT which indicated that the layer structure still remained after hydrothermal treatment. And the modification draw a slight effect on the BET surface except for Hy-100-MMT. The decrease of Hy-100-MMT
BET surface was probably due to the collapse of the MMT structure under harsh hydrothermal environment as shown in Table 1. The pore size decreased after modification because the interlayer space was occupied by the modification agent in consistence with XRD results. However, the pore volume of MMT significantly increased after modification due to the new void area and new pores formed in the interlayer by modification agents.

And the DSC thermograms of triazolone, Tri/Hy-80MMT and Tri/Hy-60-MMT were shown in Fig. 6. The fusion peak of triazolone of $80{ }^{\circ} \mathrm{C}$ disappeared after prepared into MMT indicating that triazolone distributed homogeneously in amorphous state in the pores of MMT.

Table 1. The pore structural parameters of MMT, 80-MMT, Hy80-MMT, Hy-60-MMT and Hy-100-MMT

\begin{tabular}{|c|c|c|c|}
\hline Samples & $\begin{array}{c}\text { BET surface, } \\
\mathrm{m}^{2} / \mathrm{g}\end{array}$ & $\begin{array}{c}\text { Pore volume, } \\
\mathrm{cm}^{3} / \mathrm{g}\end{array}$ & $\begin{array}{c}\text { Pore size, } \\
\mathrm{nm}\end{array}$ \\
\hline MMT & 38.8 & 0.105 & 9.22 \\
\hline 80-MMT & 40.4 & 0.191 & 3.94 \\
\hline Hy-80-MMT & 42.1 & 0.185 & 3.94 \\
\hline Hy-60-MMT & 38.8 & 0.170 & 3.94 \\
\hline Hy-100-MMT & 34.7 & 0.170 & 3.94 \\
\hline
\end{tabular}

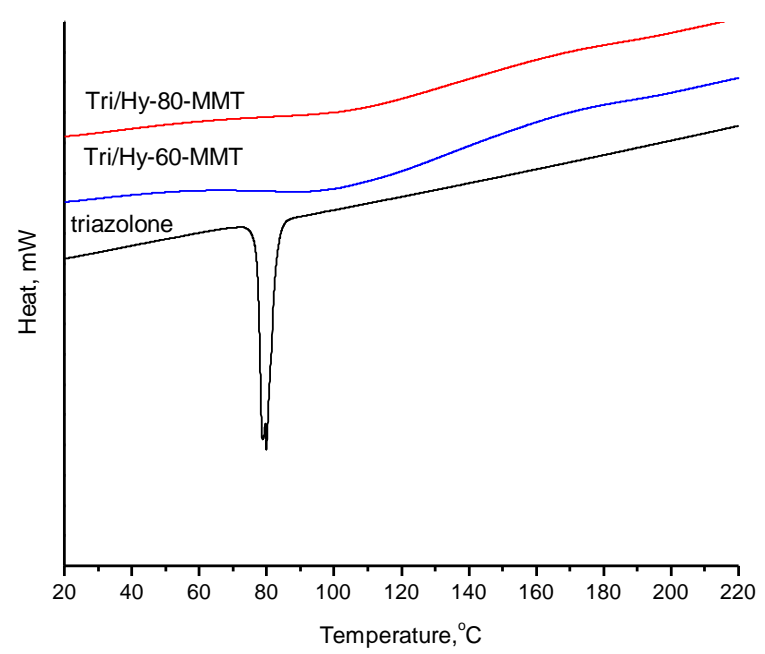

Fig. 6. DSC thermograms of triazolone, Tri/Hy-80-MMT and Tri/Hy-60-MMT

\subsection{Adsorption performance}

Table 2 listed the AC of various MMT. Hydrothermal process for preparing MMT in one step would significantly increase loading of modifying agent (SDS and KH-550) as proved by SEM results.

Table 2. The adsorption capacity of different types of MMT to triazolone

\begin{tabular}{|c|c|c|}
\hline Sample & $\begin{array}{c}\text { Hydrothermal } \\
\text { temperature, }{ }^{\circ} \mathrm{C}\end{array}$ & AC, mg/g \\
\hline Tri/MMT & - & N/A \\
\hline Tri/80-MMT & - & N/A \\
\hline Tri/Hy-S-80-MMT & 80 & N/A \\
\hline Tri/Hy-80-MMT & 80 & 19.9 \\
\hline Tri/Hy-100-MMT & 100 & N/A \\
\hline Tri/Hy-60-MMT & 60 & 29.8 \\
\hline
\end{tabular}

Therefore, the AC of MMT increased significantly after hydrothermal treatment at $60{ }^{\circ} \mathrm{C}$. The $\mathrm{AC}$ of Tri/Hy60-MMT increased up to $19.9 \mathrm{mg} / \mathrm{g}$ comparing with 
Tri/MMT and Tri/80-MMT. Tri/Hy-S-80-MMT also showed no adsorption capacity towards triazolone which further convinced that SDS and KH-550 cannot intercalate into MMT layer structure successfully without the high pressure from hydrothermal process. While for Tri/Hy-60MMT, mild preparation conditions could prevent the failure of SDS and KH-550 and collapse of the pore structure as illustrated in the SEM images. As a result, Tri/Hy-60-MMT has the best AC (29.8 mg/g) among all the samples.

\subsection{Sustained release performance}

Fig. 7 shows the sustained release performance of Tri/Hy-80-MMT with a sequence of $\mathrm{pH}=7>\mathrm{pH}=3>\mathrm{pH}=5>\mathrm{pH}=9$ in release speed. Under weak acid environment, hydrogen ion reacted with amino group from KH-550 to form ammonium group, which is positive charge. And its interaction with the negative charge SDS was strengthened. Under weak basic environment, quaternary ammonium salt was formed for triazolone with positive charge, which also strengthened the interaction with negative charge SDS. Under strong acid environment, the release speed become faster because the ionic exchange between MMT and environment was strengthened. As a result, the SDS and KH-550 inside MMT layer structure was replaced by hydrogen ion leading to the increase of hydrophilicity on the surface and the release speed was accelerated. The results proved that the system was $\mathrm{pH}$ sensitive with various sustained release performance at different $\mathrm{pH}$ value. The results indicated that the sustained release system can be well stored in weak acid or basic environment and activated while sprayed into earth or plants under neutral condition.

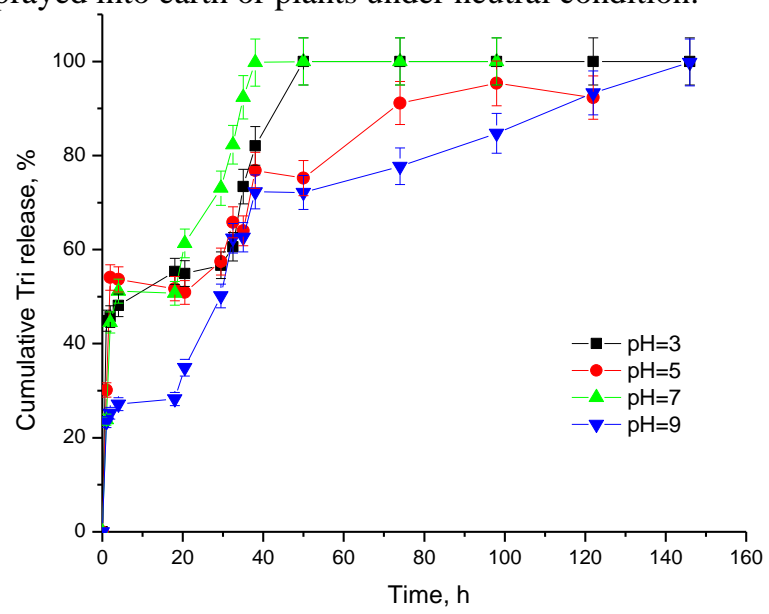

Fig. 7. Effect of various $\mathrm{pH}$ on sustained release performance of Tri/Hy-80-MMT

By now, the complete modification procedures for MMT in hydrothermal treated process and normally treated process was generally illustrated in consideration of the characterization results as shown in Fig. 8. The MMT layer structure was still maintained after hydrothermal treatment confirmed by XRD and $\mathrm{N}_{2}$ adsorption/desorption isotherms analysis. And the high pressure during hydrothermal treatment pushed the organic modification agent into the layer structure leading to the successful intercalation of SDS and KH-550 into interlayer space demonstrated by SEM and TEM images. However, the harsh react condition would cause the collapse of the layer structure and the failure of the organic modification agent. Therefore, under mild hydrothermal conditions, Tri/Hy-60MMT presented larger BET surface, pore volume and pore size has the best AC $(29.8 \mathrm{mg} / \mathrm{g})$ among all the samples. What's more, the as-synthesized system also showed significant $\mathrm{pH}$ sensitivity with a sequence of $\mathrm{pH}=7>\mathrm{pH}=3>\mathrm{pH}=5>\mathrm{pH}=9$ according to their sustained release performance.

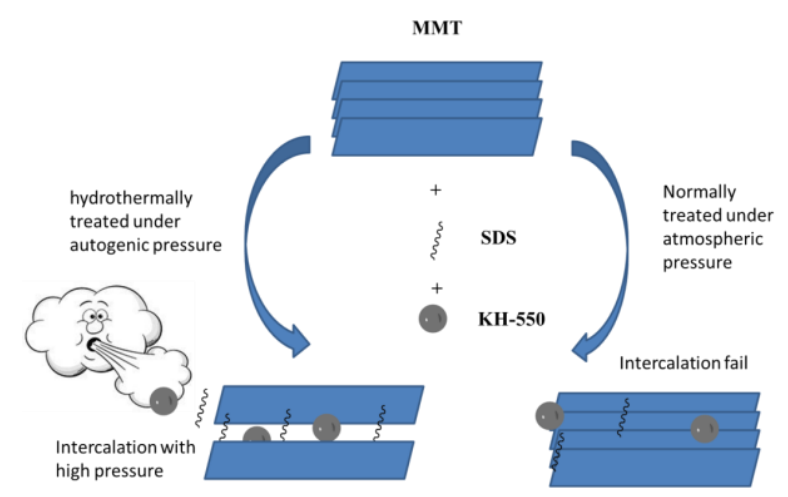

Fig. 8. Schematic illustration of the modification procedures for MMT

\section{CONCLUSIONS}

In conclusion, $\mathrm{pH}$-sensitive sustained release system of Hy-MMT was prepared through assist of the hydrothermal process, which pushed the organic modification agent into the layer structure of MMT. Triazolone existed as amorphous state homogeneously loaded on MMT, and the layer structure of the material was well-maintained after hydrothermal treatment. Hy-60MMT under mild hydrothermal treatment presented lthe best adsorption capacity $(29.8 \mathrm{mg} / \mathrm{g})$ comparing with Tri/MMT and Tri/80-MMT showing no adsorption capacity towards triazolone. The as-synthesized system showed significant $\mathrm{pH}$ sensitivity in sustained release process. Shortly, this low-cost sustained release system with $\mathrm{pH}$ sensitive is expected to have tremendous potentiality in application of controllable pesticide delivery with different sustained release behaviour at different $\mathrm{pH}$ value.

\section{Acknowledgments}

This research was supported by National Natural Science Foundation of China (Grant No. 21606262), Natural Science Foundation of Guangdong Province (No. 2017A030311003), Science and Technology Program of Guangzhou, China (No. 201707010473, 201704020025), Ordinary University Innovation Project of Guangdong Province (Grant No. 2016KTSCX062).

\section{REFERENCES}

1. Kang, W., Huang, G., Geng, Q., Hou, W., Yao, Y., Xu, B., Xing, B., Zhang, C. Effect of De-Ashing Strategies on Pore Structure and Electrochemical Performance of Activated Carbons for Supercapacitors Materials Science (Medžiagotyra) 24 (3) 2018: pp. 247-252.

https://doi.org/ 10.5755/j01.ms.24.3.18505 
2. Schmack, R., Eckhardt, B., Koch, G., Ortel, E., Kraehnert, R. ZnO Coatings with Controlled Pore Size, Crystallinity and Electrical Conductivity Materials Science (Medžiagotyra) 22 (1) 2016: pp. 74-81.

https://doi.org/10.5755/j01.ms.22.1.8634

3. Mishra, A.K., Allauddin, S., Narayan, R., Aminabhavi, T.M., Raju, K.V.S.N. Characterization of Surface-modified Montmorillonite Nanocomposites Ceramics International 38 (2) 2012: pp. 929-934. https://doi.org/10.1016/j.ceramint.2011.08.012

4. Hu, X.L., Huang, Y.P., Zhang, A.Q., Zhao, X.R. Adsorption of Organic Pollutants by Ferric Oxide Modified Montmorillonite Advanced Materials Research 955-959 2014: pp. $80-83$.

https://doi.org/10.4028/www.scientific.net/amr.955-959.80

5. Radian, A., Fichman, M., Mishael, Y. Modeling Binding of Organic Pollutants to A Clay-polycation Adsorbent Using Quantitative Structural-activity Relationships (QSARs) Applied Clay Science s116-117 2015: pp. 241-247. https://doi.org/10.1016/j.clay.2015.03.021

6. Gu, X.H., Zhou, J.L., Li, X.S., Xu, B.Y. Preparation and Research of Coupling Agent Modified Organophilic Montmorillonite Advanced Materials Research 634-638 (1) 2013: pp. 2238-2241.

https://doi.org/10.4028/www.scientific.net/amr.634638.2238

7. Ladol, J., Khajuria, H., Singh, R., Kumar, V., Sheikh, H.N. Organic Additive Assisted Hydrothermal Synthesis and Photoluminescence Properties of $\mathrm{CeF}_{3}$ : $\mathrm{Tb}^{3+}$ and $\mathrm{NaCeF}_{4}: \mathrm{Tb}^{3+}$ Nanoparticles Journal of Materials Science Materials in Electronics 28

2017: pp. $11671-11681$. https://doi.org/10.1007/s10854-017-6970-y

8. Branković, Z., Branković, G., Počuča-Nešić, M., Stanojević, Z.M., Žunić, M., Golić, D.L., Tararam, R., Cilense, M., Zaghete, M.A., Jaglicic, Z., Jagodic, M., Varela, J.A. Hydrothermally Assisted Synthesis of $\mathrm{YMnO}_{3}$ Ceramics International 41 (10) 2015: pp. 14293-14298. https://doi.org/10.1016/j.ceramint.2015.07.060

9. Ai, L., Li, L. Efficient Removal of Organic Dyes from Aqueous Solution With Ecofriendly Biomass-derived Carbon@Montmorillonite Nanocomposites by One-step Hydrothermal Process Chemical Engineering Journal 223 (3) 2013: pp. 688-695.

https://doi.org/10.1016/j.cej.2013.03.015

10. Yang, G., Jiang, Y., Yang, X., Xu, Y., Miao, S., Li, F. The Interaction of Cellulose and Montmorillonite in A Hydrothermal Process Journal of Sol-Gel Science and Technology 82 (3) 2017: pp. 846-854. https://doi.org/10.1007/s10971-017-4365-4

11. Chen, H., Huang, G., Zhou, H., Zhou, X., Xu, H. Highly Efficient Triazolone/Metal Ion/Polydopamine/MCM-41 Sustained Release System with pH Sensitivity for Pesticide Delivery Royal Society Open Science 5 (7) 2018: pp. 180658. https://doi.org/10.1098/rsos.180658

12. Rashidzadeh, A., Olad, A., Salari, D., Jalil Hejazi, M. On the Encapsulation of Natural Pesticide Using Polyvinyl Alcohol/Alginate-Montmorillonite Nanocomposite for Controlled Release Application Polymer Engineering \& Science 54 (12) 2015: pp. 2707-2714. https://doi.org/10.1002/pen.23823

13. Xu, S.M., Zhang, S.T., Shi, W.Y., Ning, F.Y., Fu, Y., Yan, H. Understanding the Thermal Motion of the Luminescent Dyes in the Dye-surfactant Cointercalated ZnAl-layered Double Hydroxides: A Molecular Dynamics Study Rsc Advances 4 2014: pp. 47472-47480. https://doi.org/10.1039/c4ra08299k

14. Antonious, G.F., Turley, E.T., $\quad$ Abubakari, M., Snyder, J.C. Dissipation, Half-lives, and Mass Spectrometric Identification of Chlorpyrifos and Tts Two Metabolites on Field-grown Collard and Kale Journal of Environmental Science \& Health Part B 52 (4) 2017: pp. $251-255$. https://doi.org/10.1080/03601234.2016.1270683

15. Wu, X., Sun, S., Wang, B., Cheng, J. The Base-Promoted Annulation of 2-Hydrazinyl Pyridine and $\mathrm{CO} 2$ toward Triazolones Advanced Synthesis \& Catalysis 359 (21) 2017: pp. :3855-3859 https://doi.org/10.1002/adsc.201700869

16. Wang, J., Mao, Z. Modified Montmorillonite and Its Application as A Flame Retardant for Polyester Journal of Applied Polymer Science 131 (1) 2013: pp. 1-15. https://doi.org/10.1002/app.39625

17. Yuan, R., Wu, S., Wang, H., Hu, L., Zhu, Y., Gao, S., Zhu, Y., Zhang, X. Facile Fabrication Approach for A Novel Multifunctional Superamphiphobic Coating Based on Chemically Grafted Montmorillonite $/ \mathrm{Al}_{2} \mathrm{O}_{3}$ Polydimethylsiloxane Binary Nanocomposite Journal of Polymer Research 24 (4) 2017: pp. 59-59. https://doi.org/10.1007/s10965-017-1222-7

18. Yang, G., Jiang, Y., Yang, X., Xu, Y., Miao, S., Li, F. The Interaction of Cellulose and Montmorillonite in A Hydrothermal Process Journal of Sol-Gel Science and Technology 82 (3) 2017: pp. 846-854. https://doi.org/10.1007/s10971-017-4365-4

19. Chen, H., Lin, Y., Zhou, H., Zhou, X., Gong, S., Xu, H. Synthesis and Characterization of Chlorpyrifos/copper(ii) Schiff Base Mesoporous Silica with $\mathrm{pH}$ Sensitivity for Pesticide Sustained Release Journal of Agricultural and Food Chemistry 64 (43) 2016: pp. 8095-8102. https://doi.org/10.1021/acs.jafc.6b03262

20. Zhou, M., Liu, Q., Wu, S., Gou, Z., Wu, X., Xu, D. Starch/chitosan Films Reinforced with Polydopamine Modified MMT: Effects of Dopamine Concentration Food Hydrocolloids 61 2016: pp. 678-684. https://doi.org/10.1016/j.foodhyd.2016.06.030

21. Yang, C., Zhang, Q., Li, J., Gao, R., Li, Z., Huang, W. Catalytic Activity and Crystal Structure Modification of $\mathrm{Pd} / \gamma-\mathrm{Al}_{2} \mathrm{O}_{3}-\mathrm{TiO}_{2}$ Catalysts with Different $\mathrm{Al}_{2} \mathrm{O}_{3}$ Contents Journal of Energy Chemistry 25 (3) 2016: pp. 375-380. https://doi.org/10.1016/j.jechem.2016.01.011

22. Zhang, Y., Shao, Y., Zhang, T., Meng, G., Wang, F. High Corrosion Protection of a Polyaniline/Organophilic Montmorillonite Coating for Magnesium Alloys Progress in Organic Coatings 76 (5) 2013: pp. 804-811. https://doi.org/10.1016/j.porgcoat.2013.01.008

23. Liu, F, Miao, L. Wang, Y. Xue, X., Yang, H. Green Fabrication of Ultraviolet Curable Epoxy Acrylate-silica Hybrid Coatings Progress in Organic Coatings 109 2017: pp. $38-44$. https://doi.org/10.1016/j.porgcoat.2017.04.015 